\title{
Phospholipid Synthesis in Lung Slices from Fetuses of Alloxan Diabetic Rabbits
}

\author{
VÂN DEMOTTAZ, MICHAEL F. EPSTEIN, AND IVAN D. FRANTZ III \\ Department of Pediatrics, Harvard Medical School, Boston, Massachusetts, USA
}

\section{Summary}

To investigate the effect of maternal diabetes on pulmonary phospholipid metabolism, we studied fetuses of rabbit does made diabetic with alloxan. Incorporation of radiolabeled choline into total and saturated phosphatidylcholine was measured in vitro in lung slices from 27.5 days gestation fetuses. No difference in choline incorporation was found between the diabetic and control groups even when disaturated phosphatidylcholine was measured. It was concluded that the previously described physiologic differences (i.e., decreases in lung deflation stability and surface activity of lung lavage) between fetuses of alloxan-diabetic and control rabbits must be due to some mechanism other than impaired phosphatidylcholine biosynthesis by the choline incorporation pathway.

\section{Speculation}

The increased occurrence of respiratory distress syndrome (RDS) in infants of diabetic mothers (IDM) may be due to a deficiency of a component of surfactant other than phosphatidylcholine, an impairment of surfactant secretion, or the presence of a surfactant inhibitor.

RDS occurs with increased frequency in the newborn IDM. Although prematurity and delivery by cesarean section are major factors in the increased incidence of RDS in IDM, Robert et al. (1) have shown that maternal diabetes mellitus itself has a significant effect on fetal lung development. There is a 5.6-fold increase in the incidence of RDS in prematurely born IDM's, when gestational age, route of delivery, and other potentially confounding factors are controlled.

Infants with RDS have unstable alveoli and progressive atelectasis as a result of the deficiency of pulmonary surfactant. The principal component of the surfactant is phosphatidylcholine (PC) (lecithin). In the lung, the PC is characterized by the presence of two saturated fatty acids esterified to the carbon atoms of the glycerol backbone, thus its designation as disaturated phosphatidylcholine or DSPC (2). Because the development of surface activity in fetal lung largely depends on biosynthesis of PC and DSPC, we examined the influence of maternal diabetes on fetal lung development by comparing the incorporation of radiolabeled choline into PC and DSPC in lung tissue from normal rabbit fetuses and fetuses of diabetic rabbit does.

\section{MATERIALS AND METHODS}

New Zealand white rabbits were mated such that gestation was known to the nearest hour. Twenty-four hr after mating, $100 \mathrm{mg} /$ $\mathrm{kg}$ of alloxan or a similar volume of saline was injected iv into the does. Twenty-four does received alloxan and 18 either died shortly after injection or aborted before delivery. The six remaining does appeared to be healthy, though polyphagic and polydipsic. The fetuses of the six alloxan-treated does were compared to those of five saline-treated does. Maternal blood glucose concentrations were monitored weekly with Dextrostix and the Ames Eyetone reflectance meter.

At 27.5 days of gestation, the fetuses were delivered by hysterotomy after killing the doe with intravenous pentobarbital. The fetuses were delivered with membranes intact and injected with ip pentobarbital to prevent breathing and expansion of the lungs with air. The fetuses were removed from their sacs and weighed. The lungs were dissected and placed in $0.9 \% \mathrm{NaCl}$ at $4^{\circ}$. A StadieRiggs microtome was used to prepare a single $0.5 \mathrm{~mm}$ slice from the right lung, including all lobes. The mean weight of the slices was $130 \pm 31(\mathrm{SD}) \mathrm{mg}$.

As a measure of the fetal lung's ability to synthesize PC and DSPC, the incorporation of radiolabeled precursor into product via the choline pathway was assayed using the technique previously described by Epstein and Farrell (3). Briefly, lung slices were preincubated in $4 \mathrm{ml}$ Krebs-Ringer-bicarbonate solution, $\mathrm{pH}$ 7.4, at $37^{\circ} \mathrm{C}$ under $95 \% \mathrm{O}_{2}, 5 \% \mathrm{CO}_{2}$ for $10 \mathrm{~min}$ in a Dubnoff metabolic shaker. Two $\mu \mathrm{Ci}$ of ${ }^{3} \mathrm{H}$-choline-chloride $(5 \mathrm{Ci} / \mathrm{mmole})$ were added to each flask, and the incubation was continued for 1 hr. The reaction was stopped by placing the flasks in an ice bath. Lipids were extracted by the method of Folch et al. (4). The tissue was homogenized in 20 volumes of chloroform-methanol (2:1) and the homogenate was filtered. One $\mathrm{ml}$ of filtrate was mixed with $0.2 \mathrm{ml}$ distilled water and the mixture centrifuged to yield two phases.

Aliquots of the upper polar phase were counted in Aquasol in duplicate. The lower apolar phase was dried under nitrogen and taken up in $1.0 \mathrm{ml}$ methanol. Duplicate $100 \lambda$ aliquots of the lipid phase were spotted on silica gel $\mathrm{H}$ plates and developed in chloroform:methanol:water (65:25:4). The lipid spots were visualized by iodination, scraped and counted in toluene-PPO-POPOP. A Searle scintillation counter was used with efficiency of $60-65 \%$. The total radioactivity measured in each PC spot was approximately 40 - to 60 -fold greater than background activity. Because previous observations in this laboratory revealed no difference between fetuses of control and diabetic mothers in lung wet weight/dry weight ratios, results were expressed as $\mathrm{cpm} / \mathrm{mg}$ wet lung/hr.

DSPC was determined by reacting duplicate $100 \lambda$ aliquots of the lower phase with osmium tetroxide and separating the disaturated lipids using neutral alumina columns (5). The eluent from the columns was chromatographed as described above. The DSPC spots were scraped and counted, and total radioactivity measured was 10- to 15 -fold greater than background. Results were expressed as $\mathrm{cpm} / \mathrm{mg}$ wet lung/hr. Recoveries were determined using labeled compounds previously purified by thin layer chromatography in this laboratory as described previously and were $82 \pm 2 \%$ for the DSPC assay and $70 \pm 2 \%$ for the PC assay. In order to eliminate the inherent variability in the amount of isotope presented to and taken up by each slice, the cpm in PC and DSPC for each fetus were adjusted as previously described (3). PC and DSPC results from each fetus within a litter were then grouped to provide a single mean value for incorporation of ${ }^{3} \mathrm{H}$-choline into ${ }^{3} \mathrm{H}$-PC and ${ }^{3} \mathrm{H}$-DSPC for each litter. The ratio of choline incorporated into DSPC and PC was calculated for each fetus. 


\section{RESULTS}

Table 1 illustrates the maternal blood glucose concentrations, fetal weights, and phospholipid synthesis results for the 11 litters studied. Maternal blood glucose concentrations at the time of killing were significantly lower in the control groups (117 \pm 5 (SE) $\mathrm{mg} / \mathrm{dl})$ than in the alloxan-treated groups, $(275 \pm 45 \mathrm{mg} / \mathrm{dl})(P$ $<0.05$ ). A total of 37 fetuses in the five control litters and 40 fetuses in the six diabetic litters were studied. The mean weight of the control fetuses was $33.5 \pm 1.5 \mathrm{~g}$ and that of the fetuses of the diabetic litters was $29.3 \pm 3.9 \mathrm{~g}$. This difference was not significant $(P<0.15)$.

The rates of incorporation of ${ }^{3} \mathrm{H}$-choline into PC and DSPC were similar in the control and alloxan-treated litters. The mean incorporation of ${ }^{3} \mathrm{H}$-choline into ${ }^{3} \mathrm{H}$-PC in the control groups was $227 \pm 34(\mathrm{SE}) \mathrm{cpm} / \mathrm{mg}$ wet lung/hr compared to $212 \pm 10 \mathrm{cpm} /$ $\mathrm{mg}$ wet lung/hr found in the fetuses of diabetic does. Incorporation of ${ }^{3} \mathrm{H}$-choline into ${ }^{3} \mathrm{H}$-DSPC was also similar in the two groups: $63 \pm 9 \mathrm{cpm} / \mathrm{mg}$ wet lung/hr in the control animals and 50 $\pm 4 \mathrm{cpm} / \mathrm{mg}$ wet lung/hr in the fetuses of diabetic does. The percent of total ${ }^{3} \mathrm{H}$-PC represented by ${ }^{3} \mathrm{H}$-DSPC was $28 \pm 2 \%$ (SE) in the control group and $23 \pm 2 \%$ in the alloxan group. Differences between diabetic and control groups were not statistically significant.

\section{DISCUSSION}

In this study, the authors utilized an animal model of the diabetic pregnancy, the alloxan-treated rabbit, to examine the effect of maternal glucose intolerance on the production of pulmonary PC and DSPC in the fetus. The activity of the major de novo pathway for the formation of surfactant phospholipids in the functional development of the fetal lung in fetuses from normal and diabetic rabbit does at 27.5 days of gestation was measured. We chose that particular gestational age because it is the critical period for maturation of fetal rabbit lung by morphologic (6), physiologic (7), biochemical (8), and survival indices (9). In addition, the interest in examining the effect of maternal diabetes on fetal lung phospholipid metabolism was stimulated by previous observations of a disparity between the physiologic and biochemical measurements of fetal lung maturity at 27.5 days of gestation in this animal model (10). Sosenko et al. (10) found that fetuses of diabetic rabbit does showed altered pulmonary pressure-volume characteristics indicative of decreased pulmonary surfactant, i.e., a significant decrease in lung deflation stability. This finding was accompanied by a decrease in the surface activity of lung lavage fluid from those fetuses. However, there were no differences in the concentration of PC and DSPC in lung tissue or in lung lavage between fetuses of normal rabbit does and those of alloxandiabetic rabbit does. The data from the current study are consistent with the biochemical findings of Sosenko et al. (10) and indicate that the maternal hyperglycemia had no significant effect on the ability of the fetal rabbit lung to incorporate choline into the major phospholipids of pulmonary surfactant, PC and DSPC.

These observations in diabetic rabbits of a disparity between physiologic and biochemical measures of pulmonary maturity in the fetus are consistent with clinical reports in diabetic human pregnancy of an incongruity between neonatal pulmonary function as manifested by RDS and the predictive biochemical index of fetal lung maturity, the amniotic fluid L/S ratio. Several investigators have observed that the incidence of RDS with a mature $\mathrm{L} / \mathrm{S}$ ratio (i.e., between $2.0-3.0$ ) is $6-20 \%$ in human diabetic pregnancies, compared to $<1 \%$ in nondiabetic pregnancies.

This abnormal relationship of amniotic fluid and pulmonary phospholipids was also observed by Epstein et al. (12) in another animal model of diabetic pregnancy, the streptozotocin-treated Rhesus monkey. They noted that the amniotic fluid $\mathrm{L} / \mathrm{S}$ ratios in the diabetic pregnancies were significantly higher than in the controls despite there being no difference in fetal lung PC concentrations. They also noted, however, an increased activity of the choline incorporation pathway in fetuses of the diabetic pregnancies. This difference between the two studies in the effect of maternal hyperglycemia on choline incorporation in diabetic animal pregnancies may be due to differences in the two animal models. In the streptozotocin-treated monkeys, maternal hyperglycemia was mild and intermittent and resulted in fetuses which were macrosomic. In contrast, the alloxan-treated rabbit does were consistently hyperglycemic to levels of $150 \mathrm{mg} / \mathrm{dl}$ and, on occasion, to greater than $400 \mathrm{mg} / \mathrm{dl}$, and the fetuses showed a tendency towards lower body weights. Thus, the differences in the effect of maternal diabetes on the rate of choline incorporation into $\mathrm{PC}$ in fetal lung may be due to the different beta cell cytotoxins, species specificity, or to differences in the severity of diabetes.

Alloxan-induced diabetes in pregnant rabbits has been well characterized by Wellman et al. (13) and in previous work in this laboratory. Wellman et al. (13) described histologic changes in the

Table 1. Effects of maternal diabetes on fetal rabbits

\begin{tabular}{|c|c|c|c|c|c|c|}
\hline Litter & $\begin{array}{l}\text { No. of } \\
\text { fetuses }\end{array}$ & $\begin{array}{c}\text { Maternal } \\
\text { Dextrostix at } \\
\text { Killing } \\
(\mathrm{mg} / \mathrm{dl})\end{array}$ & Mean fetal weight (g) & $\begin{array}{c}\text { PC } \\
(\mathrm{cpm} / \mathrm{mg} \text { lung/hr) }\end{array}$ & $\begin{array}{c}\text { DSPC } \\
\text { (cpm/mg lung/hr) }\end{array}$ & $\mathrm{DSPC} / \mathrm{PC} \%$ \\
\hline \multicolumn{7}{|l|}{ Control } \\
\hline 1 & 8 & 110 & $28.58 \pm 3.40(\mathrm{SD})$ & $357 \pm 71(\mathrm{SD})$ & $91 \pm 16(\mathrm{SD})$ & 26 \\
\hline 2 & 8 & 100 & $35.36 \pm 2.34$ & $202 \pm 48$ & $64 \pm 16$ & 32 \\
\hline 3 & 7 & 130 & $32.45 \pm 2.73$ & $179 \pm 42$ & $41 \pm 7$ & 23 \\
\hline 4 & 8 & 120 & $33.78 \pm 2.62$ & $168 \pm 19$ & $50 \pm 5$ & 30 \\
\hline \multirow[t]{2}{*}{5} & 6 & 125 & $37.41 \pm 5.00$ & $228 \pm 31$ & $68 \pm 11$ & 30 \\
\hline & 37 & $117 \pm 5(\mathrm{SE})$ & $33.52 \pm 1.48(\mathrm{SE})$ & $227 \pm 34(\mathrm{SE})$ & $63 \pm 9(\mathrm{SE})$ & $28 \pm 2(\mathrm{SE})$ \\
\hline \multicolumn{7}{|l|}{ Diabetic } \\
\hline 1 & 10 & 170 & $32.98 \pm 4.46(\mathrm{SD})$ & $224 \pm 20(\mathrm{SD})$ & $66 \pm 3(\mathrm{SD})$ & 29 \\
\hline 2 & 5 & 280 & $39.43 \pm 4.69$ & $208 \pm 36$ & $52 \pm 7$ & 25 \\
\hline 3 & 5 & 205 & $31.59 \pm 6.44$ & $200 \pm 13$ & $48 \pm 6$ & 24 \\
\hline 4 & 8 & $>400$ & $12.05 \pm 3.07$ & $236 \pm 13$ & $52 \pm 3$ & 22 \\
\hline 5 & 7 & 195 & $34.66 \pm 6.40$ & $172 \pm 12$ & $33 \pm 3$ & 19 \\
\hline \multirow[t]{2}{*}{6} & 5 & $>400$ & $25.38 \pm 1.87$ & $229 \pm 15$ & $46 \pm 2$ & 20 \\
\hline & 40 & $>275 \pm 42(\mathrm{SE})$ & $29.35 \pm 3.90(\mathrm{SE})$ & $212 \pm 10(\mathrm{SE})$ & $50 \pm 4(\mathrm{SE})$ & $23 \pm 2(\mathrm{SE})$ \\
\hline
\end{tabular}


pancreatic beta cells of rabbit pups born after alloxan-induced diabetes. These histologic changes suggested pancreatic beta cell hyperfunction. In addition, there was an increase in insulin content in the pancreas of these newborn pups. Although fetal blood glucose and insulin concentrations were not measured in the current study, unpublished observations in this laboratory have indicated that the concentration of fetal blood glucose is $86 \pm 5 \%$ (range $77-97 \%$ ) of maternal blood glucose concentration at the time of killing in both alloxan-treated and control litters. This relationship was independent of the level of maternal blood glucose over a wide range as has been found in other species (14). There was no difference in mean fetal serum insulin concentration in the two groups. Whether it is fetal pancreatic beta cell hyperplasia or insulin content, fetal serum insulin concentration, or fetal hyperglycemia which is the critical variable in determining the interaction between maternal diabetes and fetal lung maturation remains unanswered. Further studies to characterize this model are in progress.

Other investigators have utilized in vitro systems to isolate and examine the effect of insulin on fetal lung cells or explants and have described differences in choline incorporation, glycogen concentration, and the number of lamellar bodies $(15,16)$. Although these specific insulin effects are clear, their relevance to the intact fetus in situ subjected to multiple metabolic substrate and hormonal imbalances is unclear. From the current study and that of Sosenko et al. (10), the authors believe that the adverse effect of maternal diabetes mellitus on fetal lung physiologic maturation in the rabbit may not be ascribable to abnormalities in the synthesis and storage of PC and DSPC. Perhaps, as several investigators have suggested, other constituents of surfactant such as phosphatidylglycerol (17) or the lung specific apoprotein, are affected by maternal diabetes. Alternatively, as suggested by Epstein et al. (12), there may be an alteration in the secretion or release of PC and DSPC, either before or after birth, resulting in abnormal surfactant availability despite adequate synthesis and stores. Two other possible explanations for the increased incidence of RDS in IDM are the presence of an increased rate of degradation of the surfactant in alveoli or the presence of a substance which inhibits the surface activity of the surfactant film at the alveolar surface.

In summary, it has been shown that the rate of choline incorporation into PC and DSPC, the principal surfactant phospholipids, does not appear to be diminished in lung slices from 27.5 days gestation fetuses of diabetic rabbit does. Therefore, the diminished alveolar surfactant measured by Sosenko et al. (10) in the lung of the fetus of the diabetic rabbit must be explained by some other mechanism.

\section{REFERENCES AND NOTES}

1. Robert, M. F., Neff, R., Hubbell, J. P., Taeusch, H. W., and Avery, M. E. Association between maternal diabetes and the respiratory-distress syndrome in the newborn. N. Engl. J. Med., 294: 357 (1976).

2. Farrell, P. M., and Avery, M. E.: Hyaline membrane disease. Am. Rev. Resp. Dis., 111: 657 (1975).

3. Epstein, M. F., and Farrell, P. M.: The choline incorporation pathway: Primary mechanism for de novo lecithin synthesis in fetal primate lung. Pediatr. Res., 9: 658 (1975).

4. Folch, J., Lees, M., and Sloane-Stanley, G. H.: A simple method for the isolation and purification of total lipids from animal tissues. J. Biol. Chem., 226: 496 (1957).

5. Mason, R. J., Nellenbogen, J., and Clements, J. A.: Isolation of disaturated phosphatidylcholine with osmium tetroxide. J. Lipid Res., 17: 281 (1976).

6. Kikawa, Y., Kaibara, M., Motoyama, E. K., Orzalesi, M. M., and Cook, C. D. Morphologic development of fetal rabbit lung and its acceleration with cortisol. Amer. J. Pathol., 64: 423 (1971).

7. Kotas, R. V., and Avery, M. E.: Accelerated appearance of pulmonary surfactant in the fetal rabbit. J. Appl. Physiol., 30: 358 (1971).

8. Hallman, M., and Raivio, K. I.: Formation of disaturated lecithin through the lysolecithin pathway in the lung of the developing rabbit. Biol. Neonate, 27: 329 (1975).

9. Motoyama, E. K., Orzalesi, M. M., Kikawa, Y., Kaibara, M., Wu, B., Zigas, C J., and Cook, C. D.: Effect of cortisol on maturation of fetal rabbit lungs. Pediatrics, 48: 547 (1971).

10. Sosenko, I. R. S., Lawson, E. E., Demottaz, V., and Frantz, I. D.: Delayed lung maturation in fetuses of alloxan diabetic rabbits. Pediatr. Res. (Abstract), 12: 569 (1978).

11. Frantz, I. D., and Epstein, M. F.: Fetal lung development in pregnancies complicated by diabetes. Sem. Perinatol., 2: 347 (1978).

12. Epstein, M. F., Farrell, P. M., and Chez, R. A.: Fetal lung lecithin metabolism in the glucose-intolerant rhesus monkey pregnancy. Pediatrics, 57: 722 (1976).

13. Wellman, K. F., Volk, B. W., Lazarus, S. S., and Boncato, P.: Pancreatic B cell morphology and insulin content of normal and alloxan-diabetic rabbits and their offspring. Diabetes, 18: 138 (1969).

14. Chez, R. A., Mintz, D. H., Reynolds, W. A., and Hutchinson, D. L.: Maternalfetal plasma glucose relationships in late monkey pregnancy. Amer. J. Obstet. Gynecol., 121: 938 (1975).

15. Smith, B. T., Giroud, C. J. P., Robert, M., and Avery, M. E.: Insulin antagonism of cortisol action on lecithin synthesis by cultured fetal lung cells. J. Pediatr., 87: 953 (1975)

16. Gross, I., and Smith, G. J. W.: Insulin delays the morphologic maturation of fetal rat lung in vitro. Pediatr. Res. (Abstract), 11: 515 (1977).

17. Cunningham, M. D., Desai, N. S., Thompson, S. A., and Greene, J. M.: Amniotic fluid phosphatidylglycerol in diabetic pregnancies. Am. J. Obstet. Gynecol. 131: 719 (1978).

18. The authors thank Ms. Imme Hartig-Beecken for providing excellent technical assistance and Ms. Jean Cameron and Ms. Pat Spear for valuable assistance in preparation of the manuscript.

19. This research was supported in part, by a grant from the United Cerebral Palsy Research and Educational Foundation, and the William Randolph Hearst Foundation.

20. Requests for reprints should be addressed to: Ivan D. Frantz, Joint Program in Neonatology, Children's Hospital Medical Center, 300 Longwood Avenue, Boston, MA 02115.

21. Received for publication October 11, 1978

22. Accepted for publication January 30, 1979 